\title{
Preface
}

\section{Cytopathology: Diagnostic \\ Updates and Advances in Ancillary Testing}

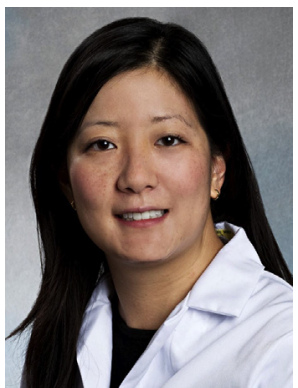

Vickie Y. Jo, MD

Editor

This issue of Surgical Pathology Clinics is devoted to cytopathology, with an emphasis on recent developments in common and challenging areas of practice. In the era of subspecialization and ancillary testing, cytopathologists remain true generalists and morphologists. However, cytologic practice now requires understanding and adapting the parallel advances in tumor classification, ancillary testing, and clinical management occurring across numerous subspecialties. Nonetheless, this is an exciting time for the field, as our ability as cytopathologists to "do more with less" with small tissue samples at the front lines of patient care is expanding greatly.

This issue provides systematic overviews and updated synopses of diagnostic criteria, ancillary testing, established and recently implemented standardized reporting schemes, and common challenges in selected areas of cytology. Covered topics include frequently encountered yet evolving areas of thyroid, gynecologic Pap smears, urine specimens, effusions, and lung cytology; challenging organ systems such as salivary gland, pancreaticobiliary, and kidney; rare and notoriously difficult bone and soft tissue tumors; and recent insights and issues with HPV-associated carcinomas of the head and neck and carcinomas of unknown primary. There are also two articles dedicated to molecular testing techniques and circulating tumor cell technology, aimed to serve as practical guides for applying these innovations to cytology.

The numerous advances in cytology enhance our diagnostic capabilities to enable accurate diagnoses and risk stratification and to detect prognostic and predictive information, which ultimately improve the care of patients with cancer. It is hoped that this collection of articles will benefit a wide audience, including cytopathologists, general pathologists, and pathologists-in-training, and will provide readers with a current and comprehensive overview of the ongoing developments in cytology and guide the integration of relevant advances (such as immunohistochemical and molecular testing) in routine practice.

Vickie Y. Jo, MD Department of Pathology Brigham and Women's Hospital and Harvard Medical School

75 Francis Street Boston, MA 02115, USA

E-mail address: vjo@partners.org 